University of Nebraska - Lincoln

DigitalCommons@University of Nebraska - Lincoln

Faculty Publications, Department of Physics and Astronomy

2011

\title{
The effective surface Debye temperature of $\mathrm{Yb}: \mathrm{GaN}$
}

S. R. McHale

J. W. McClory

J. C. Petrosky

J. Wu

R. Palai

See next page for additional authors

Follow this and additional works at: https://digitalcommons.unl.edu/physicsfacpub

This Article is brought to you for free and open access by the Research Papers in Physics and Astronomy at DigitalCommons@University of Nebraska - Lincoln. It has been accepted for inclusion in Faculty Publications, Department of Physics and Astronomy by an authorized administrator of DigitalCommons@University of Nebraska Lincoln. 
Authors

S. R. McHale, J. W. McClory, J. C. Petrosky, J. Wu, R. Palai, P. A. Dowben, and I. Ketsman 


\title{
The effective surface Debye temperature of Yb:GaN
}

\author{
S.R. McHale ${ }^{\mathrm{a}, *}$, J.W. McClory ${ }^{\mathrm{a}, * *}$, J.C. Petrosky ${ }^{\mathrm{a}}$, J. Wu ${ }^{\mathrm{b}}$, R. Palai ${ }^{\mathrm{b}, \mathrm{c}}$, P.A. Dowben ${ }^{\mathrm{c}}, \mathrm{I}$ Ketsman $^{\mathrm{c}}$ \\ a Air Force Institute of Technology, 2950 Hobson Way, Wright Patterson Air Force Base, OH 45433, USA \\ b Department of Physics and Institute for Functional Nanomaterials, University of Puerto Rico, Río Piedras, Natural Sciences Building, Phase II, San Juan, Puerto Rico, 00931, USA \\ ${ }^{c}$ Department of Physics and Astronomy, Nebraska Center for Materials and Nanoscience, University of Nebraska, P.O. Box 880111, Lincoln, NE 68588-0111, USA
}

\section{A R T I C L E I N F O}

\section{Article history:}

Received 1 November 2010

Accepted 10 February 2011

Available online 15 February 2011

\section{Keywords:}

Effective surface Debye temperature

Ytterbium doping

GaN thin films

\begin{abstract}
A B S T R A C T
The effective Debye temperature of ytterbium and gallium in Yb:GaN thin films has been obtained using X-ray photoemission spectroscopy. The vibrational motion normal to the surface results in a dimunition of photoemission intensities from which we have estimated the effective Debye temperatures of $221 \pm 30 \mathrm{~K}$ and $308 \pm 30 \mathrm{~K}$ for $\mathrm{Yb}$ and Ga, respectively. The difference between the measured values for $\mathrm{Yb}$ and Ga suggests that the Debye temperatures are influenced by the local environment. The smaller effective surface Debye temperature for $\mathrm{Yb}$ correlates to a soft, strained surface, possibly due to an increased $\mathrm{Yb}-\mathrm{N}$ bond length as compared to the $\mathrm{Ga}-\mathrm{N}$ bond length.
\end{abstract}

Published by Elsevier B.V.

\section{Introduction}

During the past decade, rare-earth doped semiconductors have generated considerable attention for their application in new optoelectronic devices [1-4]. The favorable thermal, chemical, and electronic properties of wide band gap, III-nitride semiconductors suggest device feasibility using lanthanide-doped AlN and GaN. Moreover, the tunable band gaps of these III-nitride alloys offer device applications across the visible spectrum through the ultraviolet range, to include optically stimulated lasers [5], $p-n$ junction light emitting diodes [6], and thin film electroluminescence of phosphors [7-9].

Phonon mediated exciton decay can lead to line broadening and spectral deformation. While phonons can affect excited state lifetimes, so too can the carrier concentration and local structural deformation. A signature of phonon mediated processes would be a low effective Debye temperature. The true surface Debye temperature, containing the in-plane and anharmonic motions, is difficult to measure in most surface spectroscopies [10]. However, the effective surface Debye temperature is readily obtained using X-ray photoemission spectroscopy (XPS) and ultraviolet photoemission, low energy electron diffraction, inverse photoemission spectroscopy, and other surface sensitive techniques [11-21]. The experimentally derived effective surface Debye temperature is dominated by the dynamic motion of vibrational modes normal to the surface and tends to be independent of surface orientation $[10,18]$. Increased thermal

\footnotetext{
* Principal corresponding author.

** Corresponding author.

E-mail addresses: stephen.mchale@afit.edu (S.R. McHale), john.mcclory@afit.edu (J.W. McClory).
}

vibrations dictate that the intensity of an emitted or scattered electron beam decays exponentially with increasing temperature as [11-21]

$I=I_{0} \exp (-2 W)$

where $W$ is the Debye-Waller factor and is given by

$2 W=|\Delta k|^{2}\left\langle u_{0}\right\rangle^{2}$

where $\Delta k$ is the wave vector transfer and $\left\langle u_{0}\right\rangle^{2}$ is the mean square displacement of the atoms. Within the Debye model of thermal vibrations, in the case of isotropic vibrations, $W$ is described as

$2 W=\frac{3 \hbar^{2}(\Delta k)^{2} T}{m k_{B} \Theta_{D}^{2}}$,

where $\hbar(\Delta k)$ is the electron momentum transfer, $T$ is the temperature of the sample (in Kelvin), $m$ is the mass of the scattering center, $k_{B}$ is the Boltzmann constant, and $\Theta_{D}$ is the effective surface Debye temperature. In the case of photoemission spectroscopy, the momentum transfer is equal to the momentum of the emitted electron [11-21], and the scattering center mass is the mass of the specific element from which the emitted photoelectron originates [16]. This paper reports the effective surface Debye temperatures of $\mathrm{Ga}$ and $\mathrm{Yb}$ via XPS spectra taken from Yb:GaN thin films and correlates the results with thin film strain.

\section{Experimental}

Thin films of $\mathrm{Yb}_{x} G a_{1-{ }_{x} \mathrm{~N}}(50-300 \mathrm{~nm})$ were fabricated on $\mathrm{Si}(111)$ substrates by RF plasma (EPI 620) assisted molecular beam epitaxy (MBE). The growth parameters for the deposition of Yb-doped (in situ) GaN thin films were base pressure of $\sim 10^{-11}$ Torr, nitrogen flux 
of $0.75-1.0 \mathrm{SCCM}$, RF power of $500 \mathrm{~W}$, substrate temperature of 850 $900{ }^{\circ} \mathrm{C}$, Ga cell temperature of $850^{\circ} \mathrm{C}$, and $\mathrm{Yb}$ cell temperature of $500-$ $850^{\circ} \mathrm{C}$. Thickness of the films was measured with a surface profile meter. The orientation, crystal structure, and phase purity of the films were established by $\mathrm{Cu} \mathrm{K}_{\alpha}(\lambda=1.5406 \AA)$ radiation X-ray diffraction, as shown in Fig. 1, using a Siemens D5000 X-ray diffractometer.

Surface adsorbates were removed by several preparatory sputtering and thermal annealing cycles in ultra high vacuum (UHV). The resulting surfaces were characterized by core level XPS and found to be free of oxygen. The XPS studies were performed in an UHV chamber with a hemispherical electron analyzer (Thermo VG Scientific VG100AX). The combined resolution of the system was about $1.1 \mathrm{eV}$ using a $\mathrm{Mg} K_{\alpha}$ line $(1253.6 \mathrm{eV})$ from a fixed anode $\mathrm{X}$-ray source. Surface temperature was controlled by a combination of resistive heating and cooling with liquid nitrogen. The temperature was monitored with a W-5\%Re/W-26\%Re thermocouple with an accuracy of $5 \mathrm{~K}$. The photoemission spectra were taken with a $45^{\circ}$ incidence angle and normal emission, with binding energies referenced to the Fermi level of a clean Ta foil.

\section{Results and discussion}

As anticipated, the core level photoemission intensities decrease with increasing temperature, as shown in Fig. 2(a) and (b). Fig. 3 shows the logarithm of the core peak photoemission intensities for $\mathrm{Ga}$ $3 p$ and $\mathrm{Yb} 4 d$ as a function of temperature. The kinetic energy of the outgoing photoelectron is calculated as

$E_{\text {kin }}=h \nu-\left|E_{B}\right|-\phi_{a}$,

where $h v$ is the incident photon energy, $\left|E_{B}\right|$ is the binding energy of the emitted photoelectron, and $\phi_{a}$ is the electron analyzer work function $(4.5 \mathrm{eV})$. Thus, $\Delta k$ values in Fig. 3 are calculated from

$(\Delta k)^{2}=\frac{2 m_{e} E_{k i n}}{\hbar^{2}}$,

where $m_{e}$ is the electron mass. From (1) and (3), the slope $S$ for each XPS data set in Fig. 3 determines $\Theta_{\mathrm{D}}$ from

$|S|=\frac{3 \hbar^{2}(\Delta k)^{2}}{m k_{B} \Theta_{D}^{2}}$.

The fact that the effective Debye temperature is different for ytterbium and gallium in a Yb:GaN thin film indicates that this

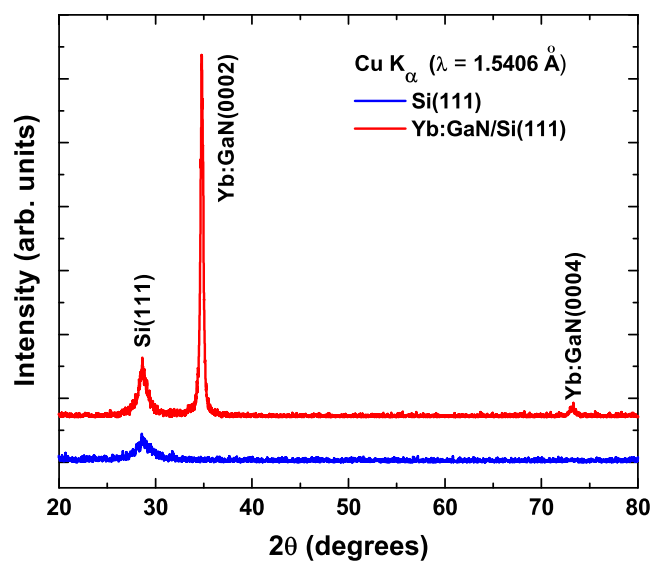

Fig. 1. The X-ray diffraction (XRD) pattern of a Yb:GaN film grown on $\mathrm{Si}(111)$ substrates. The films show c-axis orientation of the texture growth and a high level of crystallinity. The presence of any secondary phases or spurious peaks has not been observed.
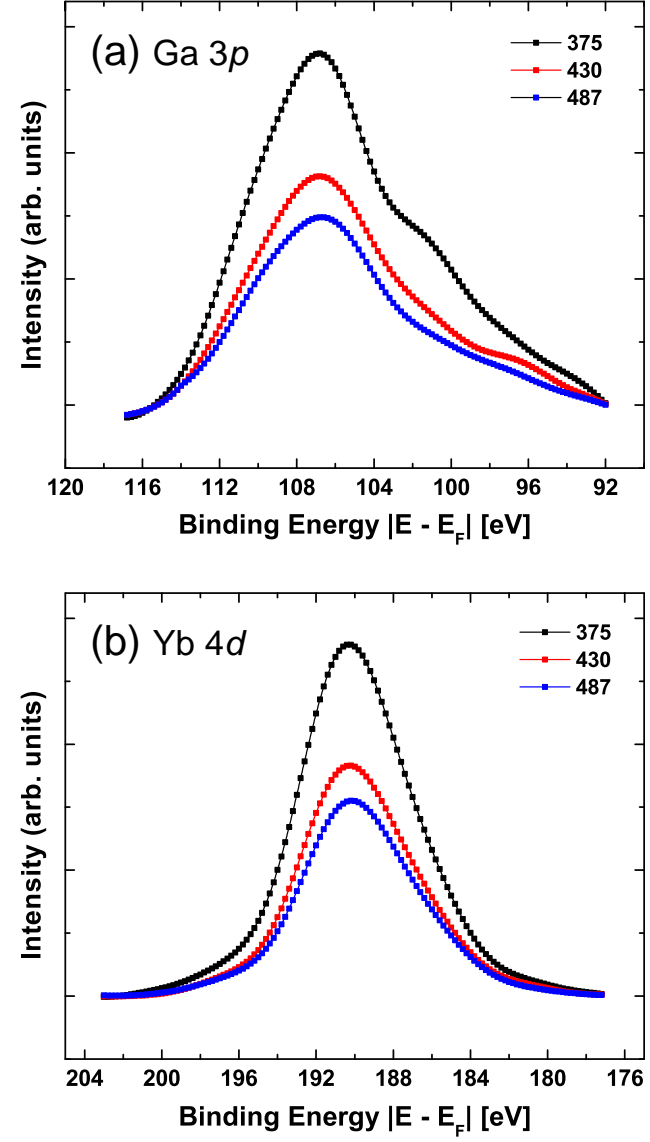

Fig. 2. Temperature dependent X-ray photoemission spectra of the indicated core levels after background subtraction.

effective Debye temperature is influenced by the local environment, not exclusively by the overall rigidity of the thin film lattice. The difference between the measured effective Debye temperatures for $\mathrm{Ga}$ and $\mathrm{Yb}$ provides an indication of the surface rigidity and lattice strain. In a simple picture, the examination of Eqs. (2) and (3) predicts an inverse relationship between atomic displacement and surface Debye temperature such that an increased $\Theta_{D}$ predicts lower atomic displacement, or a "rigid" surface. It follows that a decreased $\Theta_{D}$ is indicative of a "soft" surface. Thus, our respective values of $221 \pm 30 \mathrm{~K}$

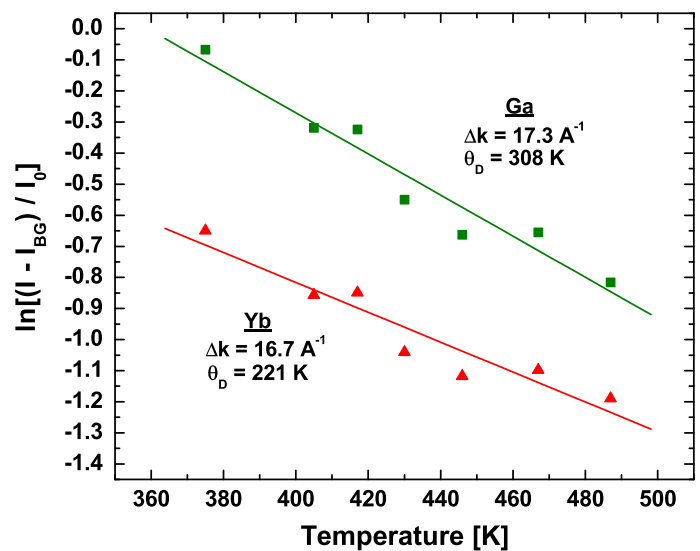

Fig. 3. Logarithm of the core peak photoemission intensities for $\mathrm{Yb}$ and $\mathrm{Ga}$ as a function of temperature following background $\left(I_{\mathrm{BG}}\right)$ subtraction and normalization to the peak intensity at the lowest temperature $I_{0}$. The experimental values were fitted (solid line) with the Debye-Waller factor. 
and $308 \pm 30 \mathrm{~K}$ for the effective $\mathrm{Yb}$ and Ga Debye temperatures in the $\mathrm{Yb}: \mathrm{GaN}$ thin film reflects softening from the introduction of $\mathrm{Yb}$ atoms into the GaN wurtzite crystal structure.

If our measured effective Debye temperatures for $\mathrm{Yb}$ and Ga were identical, within experimental uncertainty, it would be indicative of a substitutional occupation of a Ga site by a Yb ion. Although the measured effective Debye temperatures were not identical, the experimental uncertainty indicates that the $\mathrm{Yb}$ and $\mathrm{Ga}$ Debye temperatures could differ by as little as $27 \mathrm{~K}$. This similarity between the $\mathrm{Yb}$ and $\mathrm{Ga}$ Debye temperatures suggests that $\mathrm{Yb}$ doping does occur by Ga site substitution. Experimental data and crystal-field calculations have confirmed that rare-earth ions in a GaN host occupy relaxed substitutional Ga sites [1,2]. To explain the slight difference in the Debye temperatures, strain should be considered. From the standpoint of bond length, shorter bonds have higher binding energies than longer ones, which implies that decreasing the bond distance reflects stiffening and increasing bond distance reflects softening. Thus, the slightly lower calculated effective Debye temperature for $\mathrm{Yb}$ in $\mathrm{Yb}: \mathrm{GaN}$ reveals a softened surface, and we contend that this softening arises from a lengthening of the $\mathrm{Yb}-\mathrm{N}$ bond distance, as compared with the $\mathrm{Ga}-\mathrm{N}$ distance, which would manifest as thin film strain. The decrease in Debye temperature should be reflected in a decrease in exciton lifetime as this means that new phonon decay channels are opened. This in turn should lead to an increase in fluorescence peak widths.

From Fig. 1, the c-axis length of our Yb:GaN thin films was found to be approximately $5.172 \AA$, which is slightly larger than the widely reported and accepted c-axis length of undoped GaN (5.166 ̊). The XRD data are limited because the films are so very nearly crystalline, that is to say highly textured. There will be changes to the a- and b-axis lengths in response to contraction or transverse strain, as dictated by Poisson's ratio. However, the implementation of Poisson's ratio depends on the details of the stress-strain tensor, which cannot be determined accurately from our experimental data. Thus, the values for the a-axis and b-axis cannot be accurately established from our data, but would require additional measurements like grazing incidence X-ray scattering. However, the small increase in c-axis lattice constant, and hence thin film strain, can be explained due to the fact that the $\mathrm{Yb}^{3+}(8.7 \AA)$ ion has a larger ionic radius than the $\mathrm{Ga}^{3+}$ (6.2 $\AA$ ) ion. This observation suggests the substitutional doping of $\mathrm{Yb}$ ions at $\mathrm{Ga}$ sites, and further supports the claim of doping-induced thin film strain due to increased $\mathrm{Yb}-\mathrm{N}$ bond length, compared with the $\mathrm{Ga}-\mathrm{N}$ bond length.

\section{Conclusions}

X-ray photoemission spectroscopy has been used to determine the effective Debye temperatures of gallium and ytterbium in $\mathrm{Yb}$ :GaN thin films. The similarity between their measured values suggests that substitutional occupation of a Ga site by a Yb ion occurs, which supports experimental data and theoretical calculations. Careful comparison of the $\mathrm{Ga}$ and $\mathrm{Yb}$ values indicates that the slightly smaller effective Debye temperature of the $\mathrm{Yb}$ atom reflects a surface that is softened and strained, possibly due to an increased $\mathrm{Yb}-\mathrm{N}$ bond length as compared to the $\mathrm{Ga}-\mathrm{N}$ bond length.

\section{Acknowledgements}

This work was supported by the Defense Threat Reduction Agency (DTRA), the Nebraska Materials Science and Engineering Center, the Institute for Functional Nanomaterials, and NASA-IDEA-PR.

The views expressed in this article are those of the authors and do not reflect the official policy or position of the Air Force, Department of Defense, or the U.S. Government.

\section{References}

[1] Koubaa T, Dammak M, Kammoun M, Jadwisienczak WM, Lozykowski HJ. J Alloy Comp 2010;496:56-60.

[2] Koubaa T, Dammak M, Kammoun M, Jadwisienczak WM, Lozykowski HJ, Anders A. J Appl Phys 2009;106:013106.

[3] Jadwisienczak WM, Lozykowski HJ. Opt Mater 2003;23:175-81.

[4] Kenyon AJ. Prog Quantum Electron 2002;26:225-84.

[5] Park JH, Steckl AJ. Phys Status Solidi A 2008;205:26-9.

[6] Nishikawa A, Kawasaki T, Furukawa N, Terai Y, Fujiwara Y. Appl Phys Express 2009;2:1004.

[7] Steckl AJ, Heikenfeld J, Lee DS, Garter M. Mater Sci Eng, B 2001;81:97-101

[8] Tao JH, Perea-Lopez N, McKittrick J, Talbot JB, Klinedinst K, Raukas M, et al. Phys Status Solidi C 2008;5:1889-91.

[9] Shi J, Chandrashekhar MVS, Reiherzer J, Schaff W, Lu J, Disalvo F, et al. Phys Status Solidi C 2008;5:1495-8.

[10] Waldfried C, Mcllroy DN, Zhang J, Dowben PA, Katrich GA, Plummer EW. Surf Sci 1996;363:296-302.

[11] Fukutani K, Lozova N, Zuber SM, Dowben PA, Galiy P, Losovyj YB. Appl Surf Sci 2010;256:4796-800

[12] Feng DQ, Dowben PA, Rajesh R, Redepenning J. Appl Phys Lett 2005;87:181918.

[13] Jeong HK, Skomski R, Waldfried C, Komesu T, Dowben PA, Vescovo E. Phys Lett A 2004;324:242-6.

[14] Losovyj YB, Yakovkin IN, Jeong HK, Wisbey D, Dowben PA. J Phys Condens Matter 2004; $16: 4711$.

[15] Schwartz PV, Lavrich DJ, Scoles G. Langmuir 2003;19:4969-76.

[16] Jeong HK, Komesu T, Dowben PA, Schultz BD, Palmstrøm CJ. Phys Lett A 2002;302: 217-23.

[17] Borca CN, Komesu T, Jeong H, Dowben PA, Ristoiu D, Hordequin C, et al. Appl Phys Lett 2000;77:88.

[18] Borca CN, Choi J, Adenwalla S, Ducharme S, Dowben PA, Robertson L, et al. Appl Phys Lett 1999;74:347.

[19] Camillone N, Chidsey CED, Liu G, Putvinski TM, Scoles G. J Chem Phys 1991;94: 8493.

[20] Tonner BP, Li H, Robrecht MJ, Chou YC, Onellion M, Erskine JL. Phys Rev B 1986;34: 4386-9.

[21] Williams RS, Wehner PS, Stöhr J, Shirley DA. Phys Rev Lett 1977;39:302-5. 